occurred in 115 subjects; 67 of which were felt to be probably or possibly related to the intervention.

Conclusions Recruitment for a trial of pre-clinical or incomplete lupus is difficult. Barriers included perceived risk of medication for an asymptomatic condition, or desire to take medication, even if not known to work. Nevertheless, a significant number of subjects has transitioned to lupus during the course of the trial. Data comparing HCQ to placebo will be available in 2023.

Acknowledgments This trial is supported by NIH/NIAMS U01 AR071077

Trial Registration ClinicalTrials.gov NCT03030118.

\section{TELEMEDICINE IN RHEUMATOLOGY: A SURVEY OF PATIENT AND PROVIDER SATISFACTION WITH VIRTUAL CARE}

${ }^{1}$ Wei Tang, 'Leila Khalili, ${ }^{2}$ Maria Dall'Era, ${ }^{3}$ Cynthia Aranow, ${ }^{3}$ Meggan Mackay, ${ }^{1}$ Anca Askanase*. ${ }^{1}$ Columbia University Irving Medical Center, Department of Medicine, Division of Rheumatology, New York, NY; 'Lupus Clinic and Rheumatology Clinical Research Center, University of California San Francisco, San Francisco, CA; ${ }^{3}$ Feinstein Institute for Medical Research, Manhasset, NY, 11030

10.1136/lupus-2021-lupus21century.48

Abstract 1105 Table 1 Telemedicine Seven-item Questionnaire

\begin{tabular}{|c|c|c|}
\hline Questionnaire Item & Response & $\mathrm{N}(\%)$ \\
\hline \multirow{5}{*}{$\begin{array}{l}\text { How satisfied were you with your } \\
\text { previous telemedicine visit? }\end{array}$} & Highly satisfied & $50(50 \%)$ \\
\hline & Satisfied & $34(34 \%)$ \\
\hline & Neither satisfied nor unsatisfied & $11(11 \%)$ \\
\hline & Not satisfied & $5(5 \%)$ \\
\hline & Highly unsatisfied & $0(0 \%)$ \\
\hline \multirow[t]{3}{*}{ Reasons for satisfaction? } & Avoid coming into the office & $73(73 \%)$ \\
\hline & Call went smoothly & $77(77 \%)$ \\
\hline & $\begin{array}{l}\text { Decrease their concerns over } \\
\text { condition, medications and risk of } \\
\text { COVID-19 }\end{array}$ & $75(75 \%)$ \\
\hline \multirow[t]{3}{*}{ Reasons for unsatisfaction? } & Technical difficulties & $4(4 \%)$ \\
\hline & Visit was too short & $2(2 \%)$ \\
\hline & Visit was too basic for their needs & $4(4 \%)$ \\
\hline \multirow{5}{*}{$\begin{array}{l}\text { How comfortable were you with your } \\
\text { previous telemedicine visits? }\end{array}$} & Very comfortable & $62(62 \%)$ \\
\hline & Comfortable & $24(24 \%)$ \\
\hline & $\begin{array}{l}\text { Neither comfortable nor } \\
\text { uncomfortable }\end{array}$ & $11(11 \%)$ \\
\hline & Uncomfortable & $3(3 \%)$ \\
\hline & Highly uncomfortable & $0(0 \%)$ \\
\hline \multirow{5}{*}{$\begin{array}{l}\text { The physician was able to address } \\
\text { what was bothering me through the } \\
\text { telemedicine visit? }\end{array}$} & Strongly agree & $54(54 \%)$ \\
\hline & Agree & $37(37 \%)$ \\
\hline & Don't know & $5(5 \%)$ \\
\hline & Disagree & $4(4 \%)$ \\
\hline & Strongly disagree & $0(0 \%)$ \\
\hline \multirow{5}{*}{$\begin{array}{l}\text { Overall, compared to an in-person } \\
\text { visit, the telemedicine visit was? }\end{array}$} & Much better & $10(10 \%)$ \\
\hline & Better & $6(6 \%)$ \\
\hline & Same & $57(57 \%)$ \\
\hline & Worse & $25(25 \%)$ \\
\hline & Much worse & $2(2 \%)$ \\
\hline \multirow{3}{*}{$\begin{array}{l}\text { I would have a telemedicine } \\
\text { appointment in the future, if given } \\
\text { the option. }\end{array}$} & Yes & $77(77 \%)$ \\
\hline & Unsure & $14(14 \%)$ \\
\hline & No & $9(9 \%)$ \\
\hline
\end{tabular}

Background During the COVID-19 pandemic, in-person rheumatology was largely replaced by telemedicine to ensure the safety of both patients and providers. The increased pressure on the healthcare system amidst the pandemic that created a soaring number of patients has pressed an effective supplementary healthcare format for both rheumatology and other specialties. However, it remains unclear whether telemedicine, in the commonly used video-conference format, can serve as a feasible alternative to conventional in-person clinical visits while achieving comparable patient/provider satisfaction and maintaining long-term quality of care. The objective of this study was to evaluate the patient and provider experience with video-conference based virtual care thus provide further information on the prospective use of telemedicine in rheumatology practice.

Methods April-June 2020 we disseminated a seven-item questionnaire to patients with rheumatic diseases who recently attended telemedicine encounters in a video-conference format to evaluate their satisfaction and experience with the encounter. Simultaneously, we surveyed providers who recently conducted virtual care with a similar seven-item questionnaire to evaluate provider attitude towards the virtual care.

Results A total of 100 patients and 17 care providers responded to the survey. Of the 100 patients surveyed, 84 (84\%) reported high levels of satisfaction; 86 (86\%) felt comfortable with the video-conference format and 77 (77\%) expressed willingness to use telemedicine in the future; 92 (92\%) acknowledged that physicians were able to satisfactorily address the issues and concerns that prompted the visit, and $57(57 \%)$ considered the experiences very similar to the inperson clinical visits. However, of the 17 care providers surveyed, only $3(18 \%)$ expressed satisfaction with telemedicine while $14(82 \%)$ considered telemedicine visit worse than conventional in-person clinical visits.

Abstract 1105 Table 2 Demographic characteristics of study subjects

\begin{tabular}{|c|c|c|}
\hline Characteristics & Categories & $\mathrm{N}(\%)$ \\
\hline \multirow[t]{2}{*}{ Gender } & Male & $9(9 \%)$ \\
\hline & Female & $91(91 \%)$ \\
\hline \multirow[t]{6}{*}{ Age (years) } & $20-30$ & $18(18 \%)$ \\
\hline & $30-40$ & $22(22 \%)$ \\
\hline & $40-50$ & $22(22 \%)$ \\
\hline & $50-60$ & $21(21 \%)$ \\
\hline & $60-70$ & $13(13 \%)$ \\
\hline & $70-80$ & $4(4 \%)$ \\
\hline \multirow[t]{4}{*}{ Race } & White & $41(41 \%)$ \\
\hline & Black or African American & $25(25 \%)$ \\
\hline & Asian & $7(7 \%)$ \\
\hline & Hispanic & $26(26 \%)$ \\
\hline Health & Insured & $100(100 \%)$ \\
\hline insurance & Uninsured & $0(0 \%)$ \\
\hline \multirow[t]{8}{*}{ Diagnoses } & Systemic Lupus Erythematosus (SLE) & $60(60 \%)$ \\
\hline & Rheumatoid Arthritis (RA) & $7(7 \%)$ \\
\hline & Undifferentiated Connective Tissue Diseases (UCTD) & $7(7 \%)$ \\
\hline & Psoriatic Arthritis (PsA) & $5(5 \%)$ \\
\hline & Sjogren's Syndrome (SS) & $4(4 \%)$ \\
\hline & Spondylitis & $3(3 \%)$ \\
\hline & Other (Sarcoidosis, Myositis, Osteoarthritis, & $14(14 \%)$ \\
\hline & Fibromyalgia, Uveitis, Vasculitis) & \\
\hline
\end{tabular}


Conclusions Our cohort showed high rate of patient satisfaction with telemedicine healthcare. However, the relatively low healthcare provider satisfaction rate raises concern as to whether telemedicine constitutes a satisfactory alternative to conventional in-person care. Additional researches are required to investigate the feasibility of telemedicine in long-term disease activity evaluation and patient outcome measurement.

\section{PREDICTING ADVERSE PREGNANCY OUTCOMES IN WOMEN WITH SYSTEMIC LUPUS ERYTHEMATOSUS: A COMPARISON OF MACHINE LEARNING METHODS}

${ }^{1}$ Melissa Fazzari, ${ }^{2}$ Marta Guerra, ${ }^{2,3}$ Jane Salmon, ${ }^{1}$ Mimi Kim*. 'Albert Einstein College of Medicine, New York, USA; ${ }^{2}$ Hospital for Special Surgery, New York, USA; ${ }^{3}$ Weill Cornell Medical College, New York, USA

\subsection{6/lupus-2021-lupus21century.49}

Background Nearly 20\% of pregnancies in patients with Systemic lupus erythematosus (SLE) result in an adverse pregnancy outcome (APO); early identification of women with SLE who are at high risk of APO is vital. We previously derived a risk model for APO using logistic regression and data from the PROMISSE Study, a large multi-center, multiethnic/racial study of APO in women with mild/moderate SLE and/or aPL. While this highly interpretable regression model showed promising predictive performance, we sought to determine if novel and increasingly popular machine learning (ML) approaches would enhance APO risk prediction using all available predictors and potential complex relationships such as interactions or higher order terms. We compared logistic regression modeling to LASSO, a regression approach that handles high-dimensionality and correlated predictors through shrinkage of estimated coefficients, as well as several 'black box' ML algorithms. ML techniques are well-suited to highdimensional data, require no variable selection, and unlike regression-based approaches are able to explore complex relationships without explicit input by the user.

Methods We used the original PROMISSE data (41 predictor variables from 385 subjects) with APO (71/385, 18.4\%) defined as preterm delivery due to placental insufficiency or preeclampsia, fetal or neonatal death, or fetal growth restriction. Logistic regression with stepwise selection (LR-S) was compared to LASSO, random forest (RF), neural network (NN) with 2 hidden neurons, support vector machines with RBF kernel $\left(\mathrm{SVM}_{\mathrm{RBF}}\right)$, and gradient boosting (GB). To summarize discrimination we present the area under the receiver operating curve (AUC), along with sensitivity ( $\mathrm{Sn}$ ) and specificity $(\mathrm{Sp})$ at an optimal cut-point.

Results Regression based classifiers confirmed the predictors of APO identified in our previously reported model: non-white race, use of anti-hypertensive medication, low platelets, SLE disease activity, lupus anticoagulant (LAC) + , and high diastolic blood pressure (DBP). RF additionally revealed two novel interaction variables that increased APO risk: LAC+ with anti- $\beta 2$ GPI IgM, high DBP with low C3. LR-S and LASSO were observed to have similar overall discrimination (AUC $=0.75$ vs. 0.77 , table 1 ) but LASSO had higher sensitivity $(\mathrm{S} n=0.71$ vs. 0.65$)$. ML classifiers $\mathrm{RF}$ and $\mathrm{SVM}_{\mathrm{RBF}}$ had similar good performance $(\mathrm{AUC}=0.77-0.78)$, while $\mathrm{NN}$ and GB were inferior.

Conclusions Several popular ML algorithms did not provide meaningful improvements in the prediction of APO. The strong relative performance of regression-based models with
Abstract 1106 Table 1 Summary of $5 \times 10$ fold cross-validation results

\begin{tabular}{llll}
\hline Model & AUC & Sensitivity & Specificity \\
\hline LR-S & 0.75 & 0.65 & 0.78 \\
LASSO & 0.77 & 0.71 & 0.75 \\
NN & 0.71 & 0.70 & 0.61 \\
$R F$ & 0.77 & 0.77 & 0.78 \\
GB & 0.72 & 0.72 & 0.72 \\
SVM-RBF & 0.78 & 0.78 & 0.73 \\
\hline
\end{tabular}

this large and well-characterized clinical data set is notable as these models are highly interpretable, well-understood, and generally require fewer variables to generate a risk prediction. It is unlikely that complex ML algorithms with existing variables will yield superior APO predictions; new clinical and laboratory markers may improve predictions in the future.

Acknowledgments This work was supported by $\mathrm{NIH}$ grant R21 AR076612

\section{ECONOMIC EVALUATION OF HYDROXYCHLOROQUINE USE IN AN INTERNATIONAL INCEPTION COHORT}

${ }^{1}$ Megan RW Barber, ${ }^{2} Y$ van St Pierre, ${ }^{3} J o h n$ G Hanly, ${ }^{4}$ Murray B Urowitz, ${ }^{5}$ Caroline Gordon, ${ }^{6}$ Sang-Cheol Bae, ${ }^{7}$ Juanita Romero-Diaz, ${ }^{4}$ Jorge Sanchez-Guerrero, ${ }^{8}$ Sasha Bernatsky, ${ }^{9}$ Daniel J Wallace, ${ }^{10}$ David A Isenberg, ${ }^{10}$ Anisur Rahman, ${ }^{11}$ Joan T Merrill, ${ }^{12}$ Paul R Fortin, ${ }^{4}$ Dafna D Gladman, ${ }^{13}$ Ian N Bruce, ${ }^{14}$ Michelle Petri, ${ }^{15}$ Ellen M Ginzler, ${ }^{16}$ Mary Anne Dooley, ${ }^{17}$ Rosalind Ramsey-Goldman, ${ }^{18}$ Susan Manzi, ${ }^{19}$ Andreas Jönsen, ${ }^{20}$ Graciela S Alarcón, ${ }^{21}$ Ronald FVan Vollenhoven, ${ }^{22}$ Cynthia Aranow, ${ }^{22}$ Meggan Mackay, ${ }^{23}$ Guillermo RuizIrastorza, ${ }^{24} \mathrm{~S}$ Sam Lim, ${ }^{25}$ Murat Inanc, ${ }^{26}$ Kenneth C Kalunian, ${ }^{27}$ Soren Jacobsen, ${ }^{28} \mathrm{Christine}$ A Peschken, ${ }^{29}$ Diane L Kamen, ${ }^{30}$ Anca Askanase, ${ }^{1}$ Ann E Clarke*. ${ }^{1}$ University of Calgary, Alberta, Canada; ${ }^{2}$ Research Institute of the McGill University Health Center, Montreal, Canada; ${ }^{3}$ Queen Elizabeth II Health Sciences Centre and Dalhousie University, Halifax, Nova Scotia, Canada; ${ }^{4}$ Centre for Prognosis Studies in the Rheumatic Diseases, Toronto Western Hospital and University of Toronto, Toronto, Ontario, Canada; ${ }^{5}$ Rheumatology Research Group, Institute of Inflammation and Ageing, College of Medical and Dental Sciences, University of Birmingham, Birmingham, UK; ${ }^{6}$ Hanyang University Hospital for Rheumatic Diseases, Seoul, Korea; ${ }^{7}$ Instituto Nacional de Ciencias Médicas y Nutrición, Mexico City, Mexico; ${ }^{8}$ McGill University Health Centre, Montreal, Canada; ${ }^{9}$ Cedars-Sinai/David Geffen School of Medicine at the University of California, Los Angeles, USA; ${ }^{10}$ University College London, London, UK; ${ }^{11}$ Department of Clinical Pharmacology, Oklahoma Medical Research Foundation, OKlahoma City, OK, USA; ${ }^{12} \mathrm{CHU}$ de Québec -Université Laval, Québec City, Canada; ${ }^{13}$ Arthritis Research UK Epidemiology Unit, Institute of Inflammation and Repair, Manchester Academic Health Sciences Centre, the University of Manchester, and NIHR Manchester Musculoskeletal Biomedical Research Unit, Central Manchester University Hospitals National Health Service Foundation Trust, Manchester Academic Health Science Centre, Manchester, UK; ${ }^{14}$ Johns Hopkins University School of Medicine, Baltimore, Maryland, USA; ${ }^{15}$ State University of New York Downstate Medical Center, Brooklyn, New York, USA; ${ }^{16}$ Thurston Arthritis Research Center, University of North Carolina, Chapel Hill, NC, USA; ${ }^{17}$ Northwestern University and Feinberg School of Medicine, Chicago, Illinois, USA; ${ }^{18}$ University of Pittsburgh School of Medicine, Pittsburgh, Pennsylvania, USA; ${ }^{19}$ Lund University, Lund, Sweden; ${ }^{20}$ University of Alabama at Birmingham, USA; ${ }^{21}$ University of Amsterdam, Rheumatology and Immunology Center, Amsterdam, Noord-Holland, NL; ${ }^{22}$ Feinstein Institute for Medical Research, Manhasset, New York, USA; ${ }^{23}$ BioCruces Health Research Institute, Hospital Universitario Cruces, University of the Basque Country, Barakaldo, Spain; ${ }^{24}$ Emory University School of Medicine, Atlanta, Georgia, USA; ${ }^{25}$ Istanbul University, Istanbul, Turkey; ${ }^{26}$ University of California Los Angeles School of Medicine, La Jolla, California, USA; ${ }^{27}$ Copenhagen Lupus and Vasculitis Clinic, Rigshospitalet, Copenhagen University Hospital, Copenhagen, Denmark; ${ }^{28}$ University of Manitoba, Winnipeg, Manitoba, Canada; ${ }^{29}$ Medical University of South Carolina, Charleston, USA; ${ }^{30}$ Hospital for Joint Diseases, New York University Seligman Center for Advanced Therapeutics, New York, New York, USA

10.1136/lupus-2021-lupus21century.50 